Nach einem auf dem 78. Kongress der British Medical Association in London, Juli 1910, gehaltenen Vortrag.

\title{
Beiträge zur klinischen Bedeutung der papillären Kystome.
}

Von

Professor Dr. Wilhelm Nagel, Berlin.

Hierzal Tafel III.

Seit den Cutersuchungen von Wilson $\mathrm{Fox}^{1}$ ), Wald e ye $\mathrm{r}^{2}$ ), Thorton ${ }^{3}$ ) nehmen unter den Ovarialtumoren die papillären Geschwülste eine besondere Stellung ein. Denn papilläre Wucherungen bedeuten eine erhöhte Zellproliferation, und wo diese vorhanden, besteht ein gewisser Grad von Malignitat im klinischen Sinne. Die papilliren Kystome haben seit den genannten Arbeiten allerdings eine andere Klassifizierung erhalten; ihr Ursprung, ob

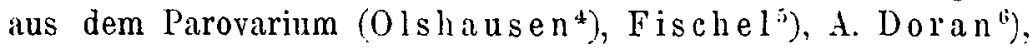
Coblenz ${ }^{7}$ ), aus dem Tubenepithel ( Marchand ${ }^{8}$ ), oder aus dem Keimepithel (Waldeyer ${ }^{n}$, Klebs ${ }^{10}$ ), deSinety, Walasse $z^{11}$ ),

1) Fox, Wils on. On the origin. struetur and mode of developement of the cystic tumours of the ovary. Med. Chir. Transaction 1864. Vol. XLVII.

$\Rightarrow$ Wald e y e r, W. Die epithelialen Eierstockgeschwülste, inshesondere die Kystome. Arch. f. Gyn., Bd. I.

3) Thorton. Transaction Obst. Soc. London 1882.

t) Olshausen. Die Krankheit der Ovaried, Stuttgart 1886 .

5) Fischel. Über Parovarialcysten und parovariale Kystome. Arch. f. Gyn., Bd. 15 .

$\left.{ }^{6}\right)$ Dor an, A. Broad Ligament Cysts above the Fallopian tube, Transact., of the patholog. Soc. of. London 1886.

5) Coblenz, H. Z. Genese und Entwicklung von Kystomen oder multiloculären Flimmerepithelkystomen der Ovarien. Virchows Archiv, Bd. 84.

8) Marchand, F. Beiträge zur Kenntnis der Ovarien-Tumoren. Habilitationsschrift, Halle 1879.

9 Waldeyer, l.c.

10) Lücke und Klebs. Beiträge zur Ovariotomie und zur Kenntnis der Abrlominalgeschwülste. Virchows Archiv, Bd. 41.

11) de Sinéty und Malassez. Sur la structure l'origine et le développement des cystes de l'ovaireș. II. Ovaire cystiques par néoformation epitheliale. Arch. de Physiol., Paris 1878 u. 1879. 
Flaischlen ${ }^{1}$ ), Witridge Williams ${ }^{2}$ ) ist noch immer nicht aufgeklärt, aber ibre anatomischen und klinischen Erscheinungen sind dieselben geblieben. Fast die Hälfte aller papillären Eierstockgeschwülste sind Adenocarcinome, die übrigen stellen nur eine besondere Art der Kystadenome dar, indem jede Gruppe von Kystadenom papilläre Wucherungen erzeugen kann.

Die papillären Kystome bilden mehr oder weniger dünnwandige, ein- oder mehrfucherige Cysten, wobei zu bedenken ist, dass jedes Eierstockskystom zu Anfang multiloculär ist (Wald eyer). Man findet die papillären Vegetationen vorwiegend innerhalb des Hauptcystenraumes, in manchen Fällen durchbrechen die Vegetationen die Cystenwand und wuchern in die Peritonealhöhle vor. Bald sind die Zotten diffus in mehr oder weniger grosser Ausdehnung über die Innenfläche der Hauptcyste verbreitet (s. Fig. 1), bald ist eine mehr oder weniger grosse Cyste vollständig ausgefüllt von den stark gewucherten vielfach verästelten zottigen Wucherungen und die Flüssigkeitsmenge ist gering. Schneidet man einen solchen Sack an, so stürzen, wie Wald e yer. so trefflich schildert, die grauweissen durchscheinenden Zottenvegetationen hervor, wie untereinander zusammenhăngende gequollene Reiskörner. Die papillären Wucherungen sind meist sehr gefăssreich und besteht ihre Grundlage aus dem zellenreichen Bindegewebe der inneren Schicht der Cystenwand. Dieselben variieren in Form und Grösse ungemein, vom einfachen feinen, schlanken Fädchen (villous growths, Fox) bis zu kurzen breiten oder hohen, vielfach verzweigten, zusammengesetzten Zotten und tragen überall einen epithelialen Überzug.

Der Cysteninhalt ist bald colloid, bald serös und enthält in einer Art der papillären Geschwülste, dem Kystadenoma pseudomucinosum papillare (Pfannenstiel), als Hauptbestandteil verschiedene Arten von Pseudomucin, welches von dem Geschwulstepithel ansgeschieden wird, und zwar, wie Waldeyer nachgewiesen hat, durch eine Metamorphose des Protoplasmas der erwăhnten Epithelzellen. In einer anderen Art der papillären Geschwülste ist der Cysteninhalt wohl serös, enthălt aber kein Pseudomucin, sondern reichlich Eiweiss, wăhrend das

1) Flaischlen, N. Zur Lehre von der Entwicklung der papillären Kystome usw. Z. f. Geburtshilfe u. Gynäkologie, Bd. 6.

?) Willia in s, W. Johns Hopkins Hospital Reports, Bd. 3, 1893. 
Epithel der Innenwand und der Papillen meist Flimmer haare trägt. (Kystadenoma serosum papillare, Pfannenstiel.) Wie man sieht, hat Pfannenstiel bei seiner Kilassifizierung sich hauptsăchlich nach der Beschaffenheit des Cysteninhaltes gerichtet. Es muss indes noch dahingestellt bleiben, ob seine Einteilung der papillären Kystome histogenetisch berechtigt ist.

Ich habe oben gesagt, dass die Entwicklungsgeschichte des papillaren Kystoms wie des Kystoms überhaupt noch in Dunkel gehüllt ist. Waldeyer führte bekanntlich die Entstehung der Kystome auf Keimepithelreste innerhalb des Eierstocks zurück. Wenn man nämlich bedenkt, dass der Aufbau des Eierstocks auf einer gegenseitigen Durchwachsung von Stroma und Keimepithel beruht, so ist es leicht, verstandlich, dass man zuweilen innerhalb des fertigen Eierstocks rundliche oder schlauchförmige Epithelmassen findet, die als Pflügersche Schlauche noch mit dem Oberflichenepithel zusammenhängen. Diese aus der embryonalen Periode des ()yariums herstammenden epithelialen Elemente, die nicht zur Bildung von Graafschen Follikeln verwendet worden sind, entwickeln sich nach Waldeye ${ }^{1}$ ) pathologisch $z u$ Kystomen.

Es ist anch denkbar, dass das Keimepithel in der Weise zu der Entstehung von Kystomen beiträgt, dass es infolge entzündlicher Auflagerungen an del Oberflache des Eierstocks ir Wucherung gerat und in das Eierstocksgewebe sich einsenkt unter Bildung von kleinen epithelialen Cysten dicht unter der Obertläche des Eierstocks. Derartige Gebilde sind u. a. beschrieben worden von Flaischlen") und von mir").

Einen nicht unwesentlichen Anteil an der Bildung der Kystone spielen ohne 7weifel die Auslaufer des Epoophoron, die man ziemlich bäufig (nach Schichele in über $30 \%$ ) nicht allein im Hilus, sondern in allen schichten des menschlichen Eierstocks bis gegen die Obertlache hin findet. Diese Ausläufer haben die Gestalt von epithelialen Schläuchen und sind, obwohl verschieden gedeutet, auch früher beschrieben, so von de Sinéty ${ }^{4}$ )

1) Waldeyer, l. c.

3) Flaischlen, l. c.

3) Nagel, W. Beitrag zur. Genese der epithelialen Eierstocksgeschwüllste. Arch. f. Gyn., Bd. 33.

t) de Sinéty und Malassez, l. c. 
und Yiasse $z^{1}$, Slaviansky ${ }^{2}$ ), F laischlen ${ }^{3}$ ), und von mir.4). Kürzlich sind sie von Bühle ${ }^{5}$ ), von Franqué $e^{6}$ ), Schichele ${ }^{7}$ ) und Rieländer ${ }^{8}$ ) eingehend behandelt worden. In den Eierstöcken verschiedener Tiere bilden, wie wir seit Köllikers grundlegenden Arbeiten wissen, die epithelialen Schlauche einen regelmässigen Befund und sind unter dem Namen „Markschläuche bekannt. Rieländer fand sie besonders in dem Eierstock von Kalb und Meerschwein stark ausgebildet.

Dass die Markschlänche aus der Crniele entstehen, ist wohl von allen Anatomen anerkannt worden, ebenso ist es wohl ganz ausser Zweifel, dass die oben erwähnten epithelialen Schläuche im Eierstock Erwachsener identisch mit den Markschläuchen sind und somit aus dem Wolffschen Körper, d. h. aus dem Epoorophon entstanden. R. Meyer ${ }^{9}$ ) verwirft allerdings diese Identität, indem er das Vorkommen von epithelialen Schläuchen verneint, das der Markschläuche aber zugibt. Einen Grund hierfür gibt er nicht an, scheint vielmehr fïlschlich anzunehmen, jemand wäre der Ansicht, dass die epithelialen Schlanche nachträglich in den fertigen Eierstock hineinwuchern. Eine solche Ansicht ist von den mir zugänglichen Autoren niemals geaussert worden, alle sind vielmehr der Ansicht, dass die Markschlüuche wïhrend der embryonalen Entwicklung in das Eierstocksgewebe hineingelangen. Wie man diesen Vorgang sich vorzustellen hat, schildert Hertwig trefflich in der 8 . Auflage seines Lehrbuches S. 443: „Wie bei den Amphibien, Reptilien, Vögeln und Säugetieren ron

1) de Sinéty und Malassez, l. c.

\#) Slawiansky. Kr. Filaments glandulaires trourés dans l'ovaire d'unc f'emme alılte. Bull. de la Soc. anatom. de Paris, 1873.

s) Elaischlen. l. c.

+) $\mathrm{Nag}$ a l, W. 1. c.

i) Bïhlel'. Beitrïge zur Kenntnis der Eibildung beim Kaninchen und Markstränge des Eierstockes beim Fuchs und Menschen. Z. f. wissenschaftl. Zoologie, Bd. 58, 1894.

c) von Franqué. Über Urnierenreste im Orarium. Z. f. (ieh. u. Gyn., Bd. 39.

7) Schichele. Die Herkunft der intraligament. Ovarialcyste. Vurhandl. d. deutschen Gesellsch. f. Gynäkologie, Bd. 11, 1906.

8) Rieländer. Das Paroophoron. Habilitationsschrift, Marburg 19014.

9) Me yer, R. Verhandlungen der Gesellschaft für Geburtshilfe und Gynäkologie za Berlin. Stuttgart 1910. 
verschiedenen Seiten beobachtet worden ist, wachsen aus dem ganz in der Nähe gelegenen Wo lf fschen Körper Epithelsprossen, die Geschlechtsstrănge der Urniere, hervor und dringen nach dem sich entwickelnden Eierstocke hin, schon zu einer Zeit, in welcher der Durchwachsungsprozess zwischen Keimepithel und Bindesubstanz eben beginnt. Sie nehmen, wie Braun für Reptilien, Hoffmann für Amphibien, Semon und Hoffmann für die Vögel nachgewiesen haben, aus dem Fpithel der Malpighischen Körperchen ihre Entstehung. An der Basis der als Leiste in die Leibeshöhle vorspringenden Anlage des Eierstocks treten sie darauf bei den Sängetieren, bei denen ibr weiteres Schicksal bisher am genauesten verfolgt ist, miteinander zu einem Netzwerk in Verbindung, schlingeln sich und wachsen den vom Keimepithel aus entstandenen Străngen und Eiballen entgegen. Wahrend nun aus den letzteren bei den Siugetieren die Rinde des Eierstocks sich entwickelt, nehmen erstere an der Zusammensetzung der späteren Marksubstanz teil und werden insofern auch als Markstränge bezeiclnet. Sie bleiben in der Nähe der Follikel solid, während die nach der Urniere zu eine Höhlung bekommen, welche von zylindrischen Zellen umgeben wird."

Angesichts dieser Tatsachen bleibt der Satz R. Meyers ${ }^{1}$ ) unverständlich, dass er das , congenitale Vordringen von Epoophorenschläuchen in das Ovarium für ganz unmöglich bält, ebensogut könnte man Nebenhodenkanalchen im Hoden suchen, zwischen beiden ist das rete testis beziehungsweise rete ovarii eingeschoben" !

Dass wirkliche Cysten aus den Markschlauchen entstehen, haben v. Franque $\left.e^{3}\right)$ und Schichele ${ }^{3}$ ) beobachtet. In dem von v. Franqué beschriebenen Fall waren die Cystchen schon dem unbewaffneten Auge sichtbar und reichten bis dicht unter die Oberflache des Eierstocks. Schichele fand an der Innenfläche solcher Cysten papilläre Wucherungen (in zwei Fällen von drei), so dass die Schlussfolgerung nicht unberechtigt erscheint, dass die papillären Kystome aus den in allen Schichten des Eierstocks vorkommenden Urnierenresten entstehen können.

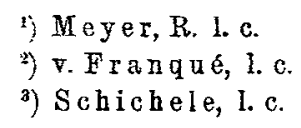


Die Häufigkeit der papillären Kystome beträgt nach Schmidtlechners ${ }^{1}$ ) \%usammenstellung aus Taufers Klinik $8,33 \%$ aller ovarialer Neubildungen und befällt häufig beide Ovarien gleichzeitig. Nach 'Tauffer ${ }^{2}$ ) war das Adenocystoma serosum papillare einseitig in $33 \%$, zweiseitig in $67 \%$ seiner Falle. Glockner ${ }^{3}$ ) ( $/$ we if els Klinik in Leipzig) fand doppelseitige Entwicklung in $60 \%$ aller Falle.

Die Aussichten auf Dauerheilung durch Operation sind keine schlechten. Von 34 Frauen mit papillärem Kystom sind nach

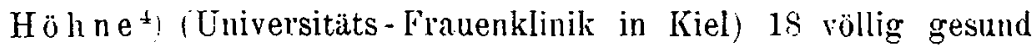
geblieben: von 45 Fälen von Adenocystoma prapillare waren б. Jahre nach der Operation 31 am Leben $=83,7 \%$ (T a uffer).

Es ist nach obigen Zahlen gewiss nicht zu leugnen, dass das papilläre Ḱystom hüufig gleichzeitig beide Ovarien befällt. Andererseits erscheint mir bei einseitiger Erkrankung die Ansicht von einer häufigen nachtraglichen Geschwulstbildung in dem zurückgelassenen gesunden Ovarium mehr auf Eindrïcken als auf bewiesenen 'Tatsachen $\mathrm{zu}$ beruhen. So entnehme ich $\mathrm{H}$ of m e i e r s ${ }^{\bar{j}}$ ! Zusammenstellung, dass bei 8 einseitigen Operationen wegen Papillom 7 gesund geblieben und nur bei einer hatte sich 1 Jahr nach der Operation wieder eine Geschwulst gebildet. 'T a u ffer $\mathrm{r}^{\text {(5) }}$ vermochte 17 Fälle ausfindig zu machen; von diesen lebten 12 über $\bar{\jmath}$ Jahre nach der Operation und nur in 2 Fälen ist die Entstehung einer Geschwulst in dem zurückgelassenen Ovarium beobachtet worden.

Weniger auffallend wird diese Tatsache, wenn man bedenkt, dass selbst bei Carcin om die nachtrigliche Erkrankung des zurückgelassenen Eierstocks nicht gerade häufig ist. Von $\mathrm{H}$ of meiers 30 Patientinnen mit einseitigem Orarialcarcinom waren

2) Sebmidtlechner, C. Prinüre und Dauerresultate der Ovariotornie bei anatom. malignen und zweifelhaften Geschwïlsten, M. f. Geb. u. Gyn., Bd. 28.

2) T a uffer. Verhandl. der deutschen Gesellsch. f. Gynäkologie, Bd. 11, Leipzig 1906.

3) Glo ckne r. Verhandl, der deutschen Gesellsch. f. Gynäkologie, Bd. 11, Leipzig 1906.

4) Höhne. ibidem.

5) Hofmeier. Verhandl. der deutschen Gesellsch. f. Gynäkologie, Bd. 11, Leipzig 1906.

') Ta uffer. ibidem. 
$15(50 \%)$ am Leben. 15 starben im Laufe der ersten 8 .Jahre nach der Operation, davon 7 an Carcinom. Ta uffer hatte von 9 Patienten mit einseitigem Ovarialcarcinom 2 wieder aufgefunden; beide waren 9 resp. 1 Jahr nach der Operation gesund.

Von From mes $\left.{ }^{1}\right) 21$ Fällen von einseitigem Ovarialcarcinom war nur in 2 die einseitige Ovariotomie ausgefïhrt worden; von diesen war die eine gestorben, während die andere noch gesund war.

Im übrigen fehlen grössere /usammenstellungen, um die Dauerresultate prozentual festzulegen, jedoch ist $\mathrm{Hofmeier}$, dem wir genaue Nachforschungen verdanken, der Ansicht, dass die Neigung des.zweiten bei deroperation noch gesunden Ovarium zu carcinomatöser Erkranknng nicht so gross ist, dass die Exstirpation auch des zweiten Orarium duchaus geboten wae.

Bei der Diskussion uber die Dauererfolge der Ovariotomie auf dern XI. Kongress deutscher (kynakologen in Kiel 190s wurden auch einige l'alle von Schwangerschaft nach einseitiger (Operation wegen Carcinom, resp, papillärem hystom bekantit gegeben, und zwar:

Hofmeier: drei Fälle ( $z$ wei bei Carcinom. einen bei papitlarem Kystomi).

T a uffer: drei Fälle (einen bei Carcinom, zwei bei papillitręm Kystom).

Fromme: einen Fall (bei papillärem Krystom).

Zu diesen möchte ich zwei Fäle aus meiner Praxis hinzufügen, die noch dadurch an Interesse gewinnen, weil sie 7 .Jahre nach der Operation beide noch völlig gesund waren.

Fall 1.2) Marie M., 37 Jahre alt.

Patientin hat fünfmal geboren, drei Kinder leben und sind gesund: vor 2 Jahren hat sie einmal abortiert. Seitdem klagt Patientin über Schmerzen hauptsächlich in der rechten Seite, ausserdem glaubt sie wahrgenommen zu haben, dass ihr Leib starker geworden ist. Ausfluss besteht nicht. Menses sind stets regelmăssig gewesen, 3 Tage dauernd, nicht profus. Patientin

1) From m e. Verhandl. der deutschen Geseilsch. f. Gynäkologie, Ba. 11, Leipzig 1906 .

3) Die beiden Füle sind beschrieben in der Dissertation ron S. Borochowitsch, Berlin 1905. 
hat kein auffallend elendes Aussehen. Die Geschwulst soll ihr nur in den letzten Monaten grössere Beschwerden verursacht haben, besonders beim Treppensteigen. Sie leidet an Verstopfung und muss öfters Urin lassen. Bei del Untersuchung konnte man von aussen einen Tumor, der sich handbreit über den Nabel fortsetzte, leicht durchfühlen. Bei der inneren Untersuchung lässt sich der Tumor vom antevertierten Uterus abgrenzen und zeigt leichte Fluktuation.

Am ō. April 1902 habe ich die Laparotomie ausgeführt. Der 'umor war mannskopfgross und gut gestielt. Mittels Troikart wurde der colloide Inhalt abgelassen, worauf der Tumor leicht in üblicher Weise entfernt wurde. Glatte Heilung.

Die Cystenwand ist von derber Beschaffenheit und mehrere Millimeter dick; an einer Stelle findet sich eine etwa walnusgrosse Cyste in die Hauptcyste eingelagert. Der Tumor steht mit der Tube durch das erhaltene Mesovarium in Verbindung, welches sich allmählich in die Oberfläche des Kystoms verliert. Vom Ovarium ist nichts mehr zu konstatieren. An der Innenfläche ist die Wand des Tumors mit reichlichen papillaren Wucherungen ausgekleidet, welche sich nicht gleichmässig ausgebreitet haben, sondern mehr an der dem Hilus zugekehrten Seite liegen (Fig. 1, Taf. III). Mikroskopisch zeigt die Cystenwand die bekannte Struktur. Die innerste Schicht besteht aus den eigentlichen Papillen, welche bald sehr schmal sind, so dass man den aus Bindegewebe bestelienden Grundstock kaum wahrnehmen kann, bald bilden sie schöne, baumartig verzweigte Zotten (Fig. 3, Tăf. III). Ausser den Zotten finden wir im Querschnitt getroffene Schläuche, welche in die Wand eingestülpt sind. Die Schläuche sind im ganzen nur in sehr geringer Zahl vorbanden und dringen nicht sehr tief in das Stroma ein.

Nach der Operation erholte die Patientin sich schnell. März 1903 blieben die Menses aus und die Geburt erfolgte am 22. Dezember leicht und glücklich; das Kind gedeibt gut.

Am 25. Juni 1910 habe ich die Patientin zuletzt untersucht: Uterus antevertiert-flectiert, nicht vergrössert, Umgebung frei, viele Faeces. Woblbefinden.

F a 11 2. Auguste B., 30 Jahre alt.

Patientin ist ein Jahr verheiratet, hat weder geboren noch abortiert. Seit einem Jahre vor der Hochzeit hat sie zeitweise 
an Schmerzen im Leibe gelitten und zwar mehr rechts. Menses alle 3-4 Wochen, durchschnittlich 6-8 Tage unter reichlichem Blutabgang, mitunter von Kreuzschmerzen begleitet. In dieser Weise ging es auch eine Zeitlang nach der Hochzeit, bis die Kreuzschmerzen heftiger wurden und Anstluss sich reichlich einstellte. Die Leibschmerzen, welche in den letzten Monaten auftraten, schildert die Patientin als gürtelförmige, krampfartige, so dass sie manchmal zu Boden stürzte. Die Schmerzen raubten ihr den Schlaf, sie hat den Appetit verloren und kam nach kurzer Zeit sehr herunter.

Patientin sieht sehr elend und anämisch aus. Der Leib ist wenig aufgetrieben. Der virginelle Uterus liegt anteflectiert, rechts ist ein 'Tumor, schwer beweglich, ron prall elastischer Konsistenz, nicht ganz kugeliger Form und Grösse eines Kindskopfs. Am 10. Marz 1902 operierte ich die Patientin. Der Tumor lag rechts, unbeweglich. Die Oberfläche war glatt, es bestand kein Ascites. Die Herausschalung des Tumols machte einige Schwierigkeiten, weil er an vielen Stellen mit dem Peritonaeum des Cavum Douglasii fest verwachsen war. Sachdem die Verwachsungen beseitigt waren, wurde der breite Stiel unterbunden und der 'Tumor von seiner Nachbarschaft abgetrennt. Das linke Ovarium erschien intakt und wurde zurückgelassen. Die Wunde heilte per primam, der Verlauf war normal. Das Allgemeinbefinden der anfänglich sehr elenden Patientin besserte sich allmählich, so dass sie nach 4 Wochen die Klinik verlassen konnte.

Schon während der Operation fiel mir die Härte des 'Tumors auf, so dass ich ein Carcinom vermutete. Der Tumor enthielt ein beschränktes Quantum von colloider Flüssigkeit, an seiner OberHache finden sich Reste der schon oben erwähnten Verwachsungen, aber keine Papillen. Seine Wand ist $1-3 \mathrm{~mm}$ dick und der einkammerige Cystenraum ist fast vollständig mit papillären Wucherungen ausgefüllt (Fig. 2, Taf. III). Am mikroskopischen Bilde fällt vor allem die reichliche Bildung von eingestülpten drüsenähnlichen Schläuchen auf, die sich hier und da verzweigen und komplizierte Gebilde darstellen (Fig. 4, Taf. III). Daneben finden sich reichliche Zotten von verschiedener Länge und Breite. Das bindegewebige Stroma ist verhăltnismåssig sebr spärlich und an vielen Stellen myxomatös degeneriert. An anderen Stellen ist es deutlich von fibrillärer Beschaffenheit und zellenreich. Das 
166 Wilhelm Nage l: Beiträge zur klinischen Bedeutung etc.

Epithel, welches das Lumen der Schlänche auskleidet, ist von zylindrischer Form und meistens einschichtig (Fig 5, Taf. III). An einigen Stellen ist das Epithel mehrschichtig; die Zellen sind hier kubisch und ragen mebr oder weniger in das Stroma hinein. Die Zotten sind durchweg mit einschichtigem Zylinderepithel bekleidet. Elimmerepithel war nirgends zu finden.

Hiernach wird man wohl geneigt sein, den Tumor als Carcinom (Adenocarcinoma papillare) anzuseben, obwohl die primäre, rein papilläre Form noch sehr deutlich ausgeprägt ist.

7wei Jahre nacl der (Operation wurde Patientin zum erstenmal schwanger und wurde am 24. Oktober 1904 glücklich von einem Madchen mittels Zange entbunden. Ich habe sie am 20. April 1909 zuletzt untersucht: Lterus in normaler Anteversion, Limgebung frei. Sie betindet sich wohl und hat nach der Operation dreimal geboren, zuletzt November 1907. Auf meine Juli 1910 an Fran B. gerichtete Anfrige erhielt ich ron dem Ehegatten die Mitteilung; dass Frau B. am 28. April 1910 einer akuten Lungenentaündung erlegen sei.

Ich schliesse aus diesen Benbachtungen, lass die unbedingte Entfernung des anderen gesunden Ovarium, wie von P'fannenstiel, Glockner und anderen verlangt wird, bei papillärem Kystom überflüssig ist. Selbst bei Carcinom ist nach Entfernung der Primärgeschwulst die gleiche Erkrankung des zurückgelassenen Ovarium nicht häufig. Ich schliesse mich deshalb $\mathrm{H}$ of meier und anderen an, dass die Gefahr einer möglicherweise wiederholten Operation bei jungen Frauen reichlich aufgewogen wird durch den Vorteil der Erhaltung des anderen Ovarium. Das wird durch die obigen Fălle von Schwangerschaft nach einseitiger Operation bewiesen.

\section{Erklärung der Abbildungen auf Tafel III.}

Fig. 1. Frau M. Papilläres Kystom (ein Drittel der natürlichen Grösse) Fig. 2. Fran B. Papilläres Kystom (Carcinom?).

Fig. 3. Schnitt durch die Cystenwand mit den Papillen (Frau M. Fall I).

Fig. 4. Schnitt durch den Zottenbanm von Fall II (Frau B.).

Fig. 5. Derselbe, starke Vergrösserung. 


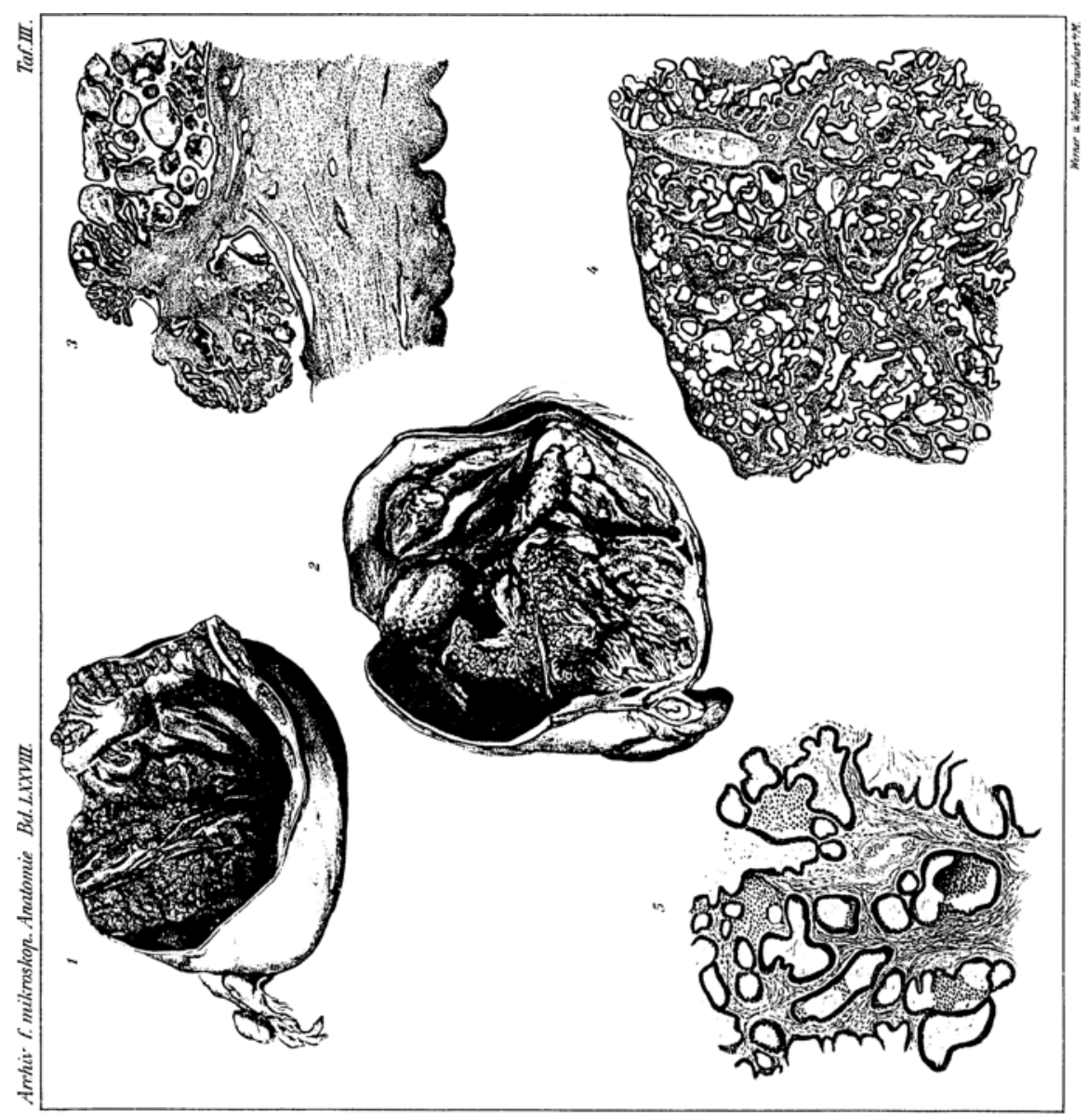

